\title{
The Association Between Hemoglobin Upswing in the Reference Range and Sleep Apnea Syndrome
}

\author{
Hirotaka Miyashita $^{1,2} \cdot$ Thomas Svensson $^{1,3,4}$ (D) Masahiro Nakamura ${ }^{1} \cdot$ Akiko Kishi Svensson $^{1,4,5}$
}

Received: 16 April 2020 / Revised: 3 June 2020 / Accepted: 9 June 2020 / Published online: 27 June 2020

(c) The Author(s) 2020

\begin{abstract}
Purpose Sleep apnea syndrome (SAS) is a relatively common disorder, but many patients with SAS are still undiagnosed. Using Japanese annual health check and medical claims data, we analyzed the association between hemoglobin upswing, defined as an increase in hemoglobin level within the reference range, and the incidence of SAS.

Methods In this study, we used the Japan Medical Database Center (JMDC) annual health check and medical claims data of 351,930 male individuals aged 40-59 who had their hemoglobin concentration checked in 2014. We initially identified the reference range of hemoglobin level based on the mean and the standard deviation of hemoglobin concentration in this population. We examined the effect of hemoglobin upswing on the incidence of SAS using Cox proportional hazards models. Results The hemoglobin upswing was defined as a change greater than $1.19 \mathrm{~g} / \mathrm{dL}$ in the reference range of 13.1 to $17.2 \mathrm{~g} /$ dL. During a mean follow-up period of approximately 1285 days, $1.9 \%$ of the individuals with hemoglobin upswing were diagnosed with SAS, while $1.6 \%$ of those without hemoglobin upswing were diagnosed with SAS. The hazard ratio of hemoglobin upswing to the incidence of SAS was 1.21 (95\% CI; $1.01-1.44, p=0.04$ ).

Conclusion We herein revealed the association between hemoglobin upswing and the incidence of SAS in a middle-aged male population. A statistically significant increase in hemoglobin concentration even in the reference range should be paid attention to as it may indicate the presence of SAS.
\end{abstract}

Keywords Sleep apnea syndrome $\cdot$ Public health $\cdot$ Annual health check $\cdot$ Hemoglobin

\begin{tabular}{|c|c|}
\hline Abbre & ations \\
\hline BMI & Body mass index \\
\hline $\mathrm{CI}$ & Confidence interval \\
\hline CPAP & Continuous positive airway pressure \\
\hline
\end{tabular}

Thomas Svensson

thomas.svensson@med.lu.se

1 Precision Health, Department of Bioengineering, Graduate School of Engineering, The University of Tokyo, 7-3-1 Hongo, Bunkyo-ku, Tokyo 113-8656, Japan

2 Graduate Medical Education, Mount Sinai Beth Israel, 281 First Avenue, New York, NY, USA

3 School of Health Innovation, Kanagawa University of Human Services, Research Gate Building Tonomachi 2-A 2 3F, 3-25-10 Tonomachi, Kawasaki-ku, Kawasaki-shi, Kanagawa 210-0821, Japan

4 Department of Clinical Sciences, Lund University, Skåne University Hospital, Jan Waldenströms gata 35, Malmö 20502, Sweden

5 Department of Diabetes and Metabolic Diseases, The University of Tokyo, 7-3-1 Hongo, Bunkyo-ku, Tokyo 113-0033, Japan

\author{
CSA Central sleep apnea \\ HR Hazard ratio \\ ICD-10 International Classification of Diseases and \\ Related Health Problems, tenth revision \\ JMDC Japan Medical Database Center \\ OSA Obstructive sleep apnea \\ SAS Sleep apnea syndrome \\ SD Standard deviation
}

\section{Introduction}

Sleep apnea syndrome (SAS) is a relatively common disorder, especially among middle-aged men. Pathophysiologically, SAS is divided into two entities; obstructive sleep apnea (OSA) which is the predominant form in the general population, and central sleep apnea (CSA). The prevalence of moderate to severe OSA and CSA in middle-aged US men is $24.0 \%$ and $0.8 \%$, respectively [1]. However, only $18.0 \%$ of middle-aged male patients with OSA have been clinically diagnosed [2]. This is due to the 
unspecific self-reported symptoms of SAS, e.g., fatigue, daytime sleepiness, and insomnia. Although severe snoring is a key component in the diagnosis of OSA, it is rarely reported without a bed partner. Without proper treatment such as continuous positive airway pressure (CPAP), OSA can increase cardiovascular morbidity and even all-cause mortality [3, 4]. Thus, more data should be accumulated to allow for better or alternative diagnostic approaches for OSA.

SAS causes hypoxemia in the body during sleep [5]. A possible consequence of systemic hypoxemia is increased level of hemoglobin which in turn could explain the high hemoglobin level in some patients with SAS [6]. Consequently, we hypothesize that the presence of a hypoxemic condition, including SAS, will result in an upswing of hemoglobin within its reference range.

\section{Methods}

\subsection{Setting}

Japanese companies are legally obliged to provide their employees with an annual health check [7]. This health check includes vital signs, laboratory data of blood and urine, and physical examination by doctors. The health check participation rate was $88.5 \%$ for annual health checks in 2012 [8].

\subsection{Data}

This study analyzed data from Japan Medical Data Center (JMDC). JMDC is a for-profit company which collects anonymized individual-level medical claims and annual health check data from several employees' health insurance schemes. JMDC assigns each individual with a random ID which in turn enables the consolidation of the medical claims and annual health check data. The information provided by JMDC includes sex, birth month, the last record (the date of the latest health check or visiting a doctor), the diagnosis, the date of diagnosis, the type of medical procedure related to SAS (continuous airway pressure and uvulopalatopharyngoplasty), and annual health check data. Diagnoses were expressed using the 2003 version of the International Classification of Diseases and Related Health Problems, tenth revision (ICD-10) [9], and medical procedures were defined in accordance with the Japanese medical claims code.

The study protocol was approved by the Institutional Review Board of the University of Tokyo (application number: 11449).

\subsection{Study Population}

JMDC data have increased in size in recent years. However, relying only on newer data could result in short follow-up times. Thus, we included individuals with hemoglobin concentration data in 2014 to ensure sufficient data size and length of the follow-up period. We then narrowed the study population to men aged between $40-59$ years as the distribution of hemoglobin concentration varies depending on age and sex [10]. Additionally, middle aged men are the most susceptible group to SAS $[11,12]$ and JMDC has a relatively large amount of data on this population as it collects data from Japanese companies. As JMDC only provides information about an individual's birth month, we calculated each individual's age based on the last day of the month of check in 2014.

Initially there were 837,110 individuals who had their hemoglobin concentration checked in 2014. We then excluded men and women of age groups other than 40-59 years, leaving 351,930 individuals (Fig. 1). Some individuals undergo health checks more than once a year because of abnormal results in the initial check of the year. Some individuals with high risk jobs (e.g., night shift or particle exposure) also have multiple health checks in one year. In these cases, we used the first annual health check of the year in our analyses. This population was analyzed for the definition of reference range of the hemoglobin level.

To analyze the association between the change of hemoglobin concentration and incidence of SAS, we included those who had their hemoglobin level examined both in 2013 and $2014(n=277,905)$. In annual health checks, hemoglobin check can be waivered for individuals whose BMI is 20 or less based on doctor's instructions. In this population, individuals with hemoglobin concentrations out of the reference range at their health check either in 2013 or 2014 were excluded $(n=18,295)$. The remaining population was examined to determine the mean and the standard deviation of the change of hemoglobin concentration within the reference range. We thereafter excluded any individuals with a diagnosis of SAS prior to the 2014 health check $(n=6143)$, and those with missing information on BMI, abdominal circumference, smoking habit, and the date of the last record $(n=7723)$. A total of 245,744 individuals were included for the analysis of the association between the main exposure (hemoglobin upswing, as described below) and the incidence of SAS. This population was also used to determine the sensitivity and specificity of the diagnostic data of SAS in the JMDC dataset.

We included 268,963 individuals who had hemoglobin checks from 2013 to 2016 in our post-hoc analyses of the 


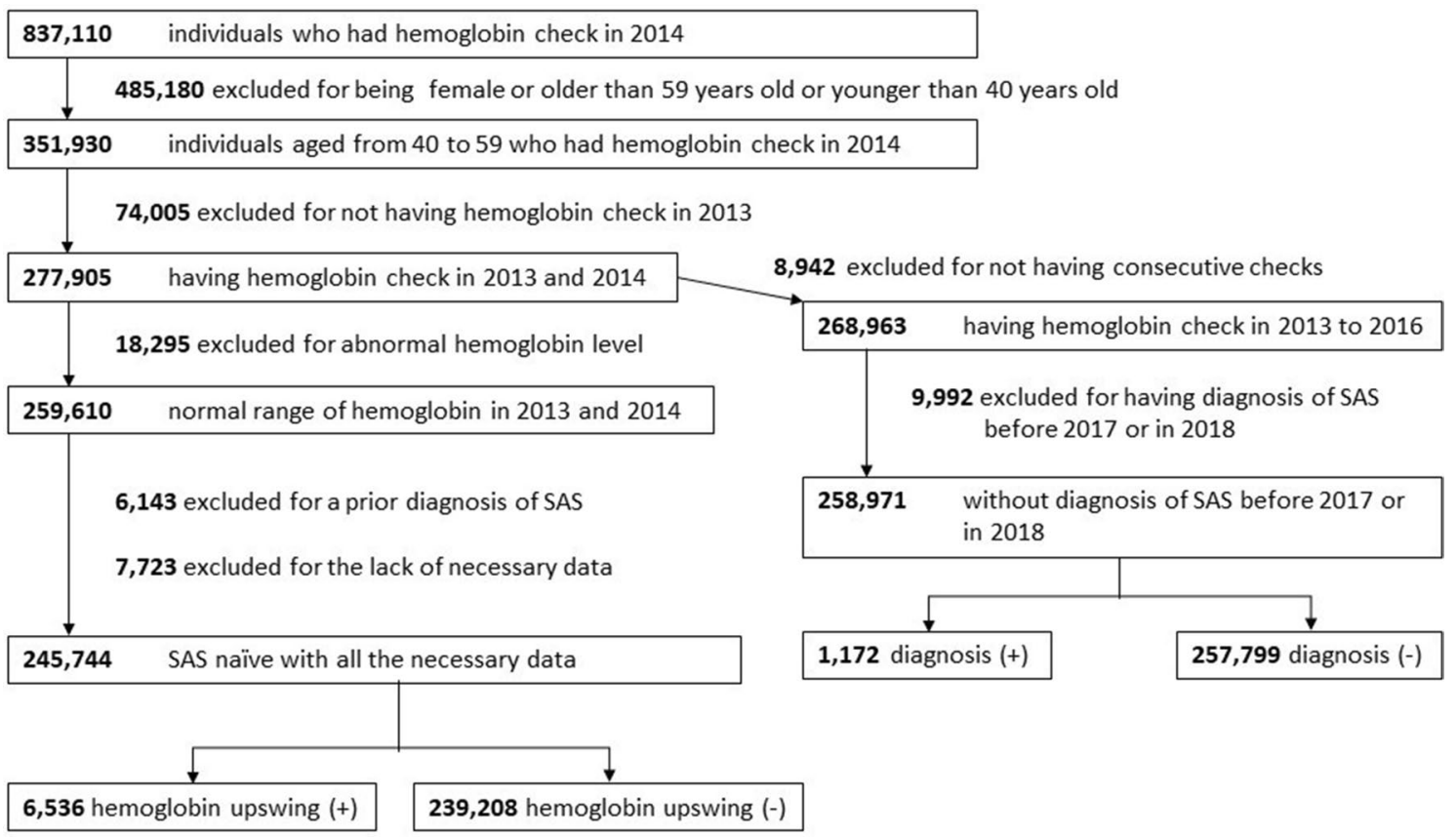

Fig. 1 Flow diagram of individuals included and excluded in the analysis

occurrence of hemoglobin upswing in individuals newly diagnosed with SAS. For these analyses, we excluded individuals who were given a diagnosis of SAS before 2017 $(n=9992)$.

\subsection{Main Exposure}

\subsubsection{Reference Range of Hemoglobin}

The reference range of hemoglobin concentration was defined as the range within two standard deviations from the mean in the study population.

\subsubsection{Hemoglobin Upswing}

The main exposure of this study was hemoglobin upswing. Hemoglobin upswing was defined as the change of hemoglobin concentration within the reference range larger than the mean plus two standard deviations.

\subsection{Sleep Apnea Syndrome (SAS)}

SAS was defined using ICD-10 code G473. We excluded any data with a suspicion flag, which suggests a suspected, but not definitive diagnosis of SAS.

\subsection{Follow-up Period}

The follow-up period of each participant was defined as the period from the date of the health check in 2014 to the date of diagnosis, or the date of the last record, whichever came first.

\subsection{Statistical Analysis}

From the annual health check data, we extracted the data concerning participants' birth month, sex, the date of the health check, hemoglobin concentration, BMI, abdominal circumference, and the answers to a question about smoking habit (i.e., smoking more than 100 cigarettes in total or for a period longer than 6 months, and having smoked cigarettes in the last month; yes/no).

We evaluated the difference of the background characteristics (mean age, the prevalence of smoking habit, mean BMI, mean abdominal circumference) of the individuals with or without hemoglobin upswing using the paired Student $t$-test for continuous variables (age, BMI, abdominal circumference), and the chi-squared test for categorical variables. Hazard ratios (HRs) and their 95\% confidence intervals (CIs) were determined in Cox proportional hazards models to characterize the relative risk of incidence of SAS from hemoglobin upswing. Model 1 was adjusted only for age, and model 2 was additionally adjusted for BMI, abdominal circumference, and smoking habit. 
We calculated sensitivity and specificity of the diagnostic data for the participants who had received treatment for SAS. We compared the number of individuals who had an initial diagnosis of SAS after their 2014 annual health check with that of individuals who initiated CPAP or underwent uvulopalatopharyngoplasty in the same period.

We also performed post-hoc analysis to calculate the sensitivity and specificity of hemoglobin upswing as a diagnostic marker for SAS. Additionally, we calculated the odds of hemoglobin upswing from 2014 to 2016 in the populations with or without the initial diagnosis of SAS in 2017. The odds ratio was evaluated using the chi-squared test.

All statistical analyses were performed using R 3.5.2 for Windows (R Foundation for Statistical Computing, Vienna, Austria). Significance levels were two-tailed and set as $\alpha=0.05$.

\section{Results}

\subsection{Study Population and the Definition of the Reference Range of Hemoglobin Level}

The mean of age of the study population was 48.4 years ( $\mathrm{SD} \pm 5.56$; range: 40 to 59 years). The mean and standard deviation of hemoglobin concentration was 15.17 and $1.04 \mathrm{~g} / \mathrm{dL}$, respectively. Consequently, the reference range of hemoglobin level in this population was defined as 13.1 to $17.2 \mathrm{~g} / \mathrm{dL}($ mean $\pm 2 \mathrm{SD})$.

\subsection{Hemoglobin Upswing}

The mean change of hemoglobin level was - 0.0386 $(\mathrm{SD} \pm 0.612) \mathrm{g} / \mathrm{dL}$. From this result, we defined hemoglobin upswing as an increase in hemoglobin concentration of more than $1.19 \mathrm{~g} / \mathrm{dL}$ (i.e., mean $+2 \mathrm{SD}$ ).

\subsection{The Impact of Hemoglobin Upswing on the Incidence of SAS}

The mean follow-up period of the 245,744 individuals included was $1285(\mathrm{SD} \pm 241)$ days. Among them, 6,536 individuals $(2.7 \%)$ had hemoglobin upswing (upswing group). Smoking was more prevalent in the upswing group $(40.6 \%$ vs. $38.6 \%, p<0.01)$. There was no significant difference in age, BMI, and abdominal circumference between the two groups (Table 1).

In the follow-up period, $1.9 \%$ of individuals in the upswing group were initially diagnosed with SAS, while the corresponding proportion in the non-upswing group was 1.6\% $(p=0.066)$ Fig. 2 shows the cumulative incidence of SAS in the two groups. In the age-adjusted model (model 1), the hazard ratio of hemoglobin upswing to the incidence of SAS was 1.20 (95\% CI; 1.00-1.43 $p=0.050$ ) (Table 2). In the multivariable adjusted model (model 2 ), the hazard

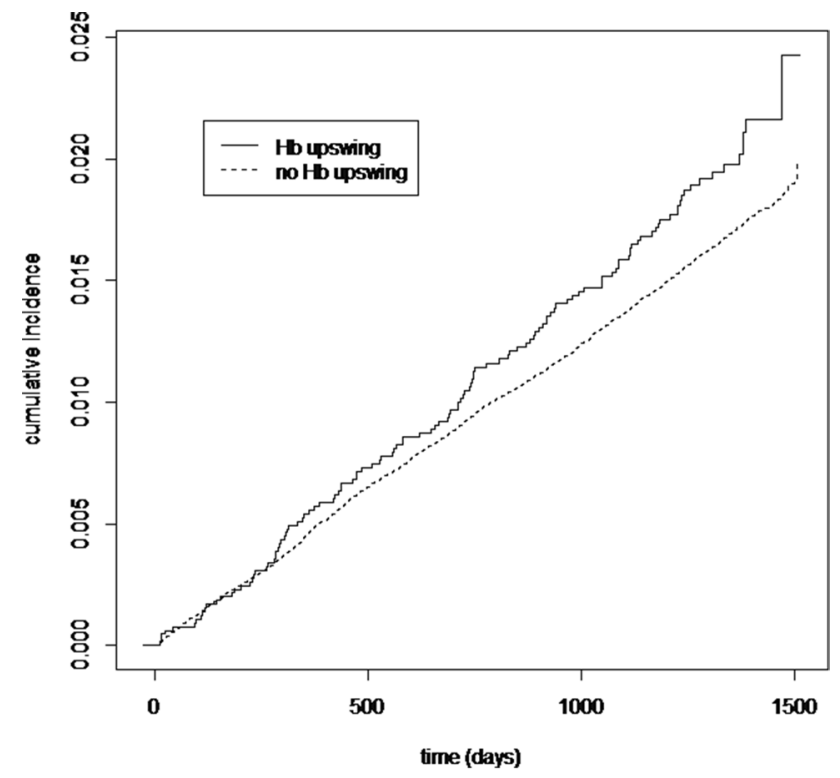

Fig. 2 Cumulative incidence of sleep apnea syndrome in the groups with or without hemoglobin upswing
Table 1 Individuals' baseline characteristics according to hemoglobin upswing

\begin{tabular}{lllc}
\hline Variables & Upswing & Without upswing & $p$ value* \\
\hline Number of individuals $(N, \%)$ & $6,536(2.66 \%)$ & $239,208(97.3 \%)$ & \\
Age $($ mean $\pm \mathrm{SD})$ & $48.5 \pm 5.49$ & $48.48 \pm 5.50$ & 0.533 \\
Smoking habit $(N, \%)$ & $2653(40.6 \%)$ & $92,292(38.6 \%)$ & $<0.01$ \\
BMI (mean kg/m $\left.{ }^{2} \pm \mathrm{SD}\right)$ & $23.7 \pm 3.33$ & $23.7 \pm 3.30$ & 0.086 \\
Abdominal circumference (mean & $83.8 \pm 8.82$ & $83.8 \pm 8.77$ & 0.835 \\
cm \pm SD) & & & 0.066 \\
Events $(N, \%)$ & $125(1.91 \%)$ & $3859(1.61 \%)$ & \\
\hline
\end{tabular}

$B M I$ body mass index

$* \chi^{2}$ test was used for categorical variables and Student's $t$-test for continuous variables 
Table 2 Hazard ratio of hemoglobin upswing on the incidence of sleep apnea syndrome

\begin{tabular}{llll}
\hline Models & Hazard ratio & $\begin{array}{l}\text { 95\% Confidence } \\
\text { interval }\end{array}$ & $p$-value \\
\hline Model 1 & 1.20 & $1.00-1.43$ & 0.050 \\
Model 2 & 1.21 & $1.01-1.44$ & $0.040^{*}$ \\
\hline
\end{tabular}

Model 1 was adjusted only for age

Model 2 was additionally adjusted for BMI, abdominal circumference, and smoking habit

Asterisk denotes $p<0.05$ with statistical significance

Table 3 The number of patients with diagnosis and treatment for sleep apnea syndrome (SAS)

\begin{tabular}{lllr}
\hline & $\begin{array}{l}\text { With SAS } \\
\text { diagnosis }\end{array}$ & $\begin{array}{l}\text { Without SAS } \\
\text { diagnosis }\end{array}$ & \multicolumn{1}{c}{ Total } \\
\hline With SAS treatments & 2017 & 9 & 2026 \\
Without SAS treatments & 1967 & 241,751 & 243,718 \\
Total & 3984 & 241,760 & 245,744 \\
\hline
\end{tabular}

ratio of hemoglobin upswing in this model was $1.21(95 \%$ CI; $1.01-1.44 p=0.040$ ).

\subsection{Evaluating the Validation of Diagnostic Data}

Of the 2026 participants who started treatment for SAS after their 2014 annual health check, 2017 (99.6\%) participants had the initial diagnosis of SAS. Among the 243,718 participants who did not receive treatment for SAS, 1967 individuals $(0.8 \%)$ had a diagnosis of SAS (Table 3$)$. From this result, the sensitivity and specificity of diagnostic data from JMDC database is $99.6 \%$ and $99.2 \%$ for SAS which requires CPAP or surgical treatment.

\subsection{Post-hoc Analysis}

Among the 258,971 participants included, 1172 individuals had an initial diagnosis of SAS in 2017, of which 85 participants $(7.3 \%)$ experienced hemoglobin upswing at least once during the 2014 to 2016 annual health checks. A total of 17,075 of $257,799(6.6 \%)$ individuals without an initial diagnosis of SAS in 2017 had hemoglobin upswing in the same period. The odds ratio was $1.10(p=0.377)$. Those diagnosed with SAS in 2017 had experienced

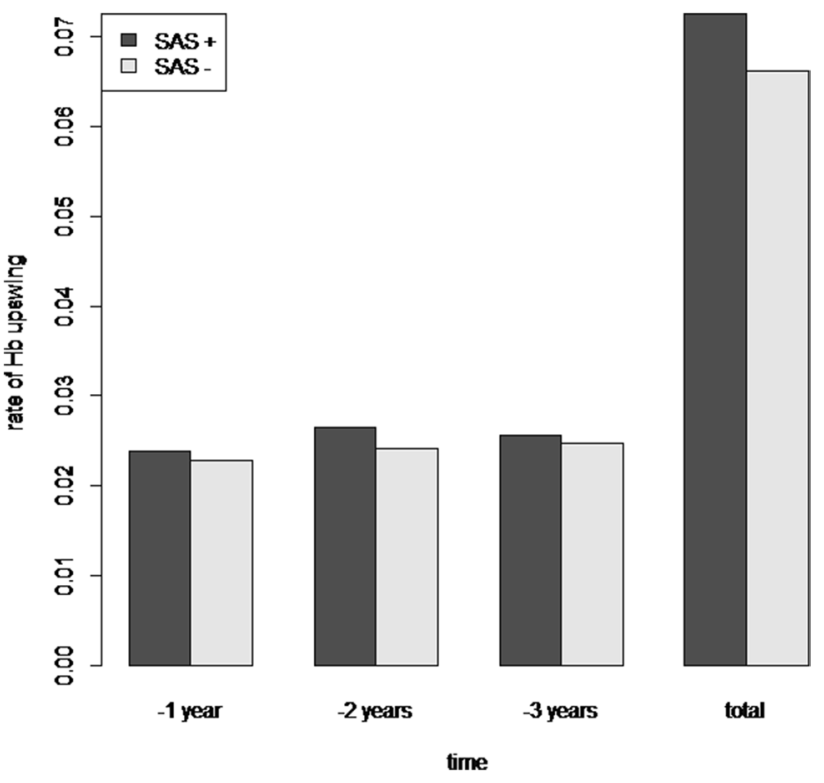

Fig. 3 The proportion of individuals experiencing hemoglobin upswing within 3 years to the initial diagnosis of sleep apnea syndrome in 2017. "1 year" is in 2016, "2 years" is in 2015 , and "3 years" is in 2014, respectively. "Total" represents the rate of individuals with hemoglobin upswing at least once in 2014 to 2016

hemoglobin upswing overall, as well as during the one, two, and three years preceding the diagnosis $(p>0.05)$ (Table 4, Fig. 3).

\section{Discussion}

This retrospective study has identified that a statistically significant hemoglobin upswing within the reference range is associated with an increased risk of incident SAS. Other findings are; the hemoglobin concentration of 13.1 to $17.2 \mathrm{~g} /$ $\mathrm{dL}$ is consistent with the range within two standard deviations from the mean in Japanese men aged 40 to 59 years. Hemoglobin upswing is associated with a higher prevalence of smoking. The sensitivity and specificity of diagnostic data in JMDC database are over $99 \%$ for SAS which requires treatment with CPAP or uvulopalatopharyngoplasty.

So far, some clinical risk factors for SAS have been identified, such as age, body mass index, male sex, snoring [13], and nocturnal choking or gasping [14]. However, no
Table 4 The proportion of individuals with diagnosis of sleep apnea syndrome (SAS) who had had hemoglobin upswing in each period $(N, \%)$

\begin{tabular}{lllll}
\hline & With SAS diagnosis & Without SAS diagnosis & Odds ratio & $p$-value \\
\hline 1 year prior & $28(2.4 \%)$ & $5889(2.3 \%)$ & 1.05 & 0.769 \\
2 years prior & $31(2.6 \%)$ & $6231(2.4 \%)$ & 1.10 & 0.568 \\
3 years prior & $30(2.6 \%)$ & $6392(2.5 \%)$ & 1.03 & 0.850 \\
Total & $85(7.3 \%)$ & $17,075(6.6 \%)$ & 1.10 & 0.377 \\
\hline
\end{tabular}


good clinical scoring system has been adopted to detect patients who require detailed examinations for sleep apnea, and only $18.0 \%$ of middle-aged male patients with OSA have been clinically diagnosed [2]. The American College of Physicians recommends polysomnography for patients with unexplained daytime sleepiness who are suspected of having SAS based on their clinical presentation [15], and survival of patients with SAS may be improved through proper treatment [4]. Our data show that the individuals who experienced hemoglobin upswing were likely to be diagnosed with SAS. Hemoglobin upswing is independent of other risk factors such as age, BMI, and abdominal circumference. The difference in the incidence of SAS between two groups (1.9\% vs $1.6 \%$ ) is not large enough to consider hemoglobin upswing as a single clue to detect SAS, but it can be an additional diagnostic tool to increase the accuracy of detecting individuals suspected of having SAS. Considering that SAS can create a hypoxemic state during sleep [5], which in turn can increase hemoglobin concentration [6], this result is reasonable and in line with our hypothesis. The variability of hemoglobin concentration has been paid attention to as a predictor of mortality in patients with end-stage renal disease [16]. To the best of our knowledge, this is the first article to focus on the effect of hemoglobin concentration change in an overall healthy population.

Additionally, we emphasized the change of hemoglobin level within the reference range by excluding individuals with abnormal hemoglobin levels. Detailed examinations are usually warranted for hemoglobin levels exceeding the reference range. However, it has been unclear whether a significant change of hemoglobin level should be further investigated. The result in this study suggests the clinical importance of a change of hemoglobin level even within the reference range.

The distribution of hemoglobin concentration varies depending on sex and ethnicity [10]. Thus, we made a specific definition of reference hemoglobin concentration in this study. We assume that the reference range of hemoglobin defined in this study, 13.1 to $17.2 \mathrm{~g} / \mathrm{dL}$, is consistent with the normal range of hemoglobin level commonly used for men at annual health checks in Japan [17].

We found that hemoglobin upswing is associated with a high prevalence of smoking. It has been shown that starting smoking can result in increased hemoglobin concentrations in male patients [18]. Additionally, in the population receiving hemodialysis, hemoglobin variability is slightly higher in current smokers [19]. We found that this association also exists in an overall healthy population.

In the post-hoc analysis, we found that $7.3 \%$ of participants with an initial diagnosis of SAS in 2017 experienced hemoglobin upswing in 2014 to 2016, whereas the corresponding proportion for individuals without diagnosis of
SAS was $6.6 \%$. This means that the sensitivity and specificity of hemoglobin upswing for new incidence of SAS in three years are $7.3 \%$ and $93.4 \%$, respectively, and that the likelihood ratio is 1.10 . Therefore, hemoglobin upswing cannot be regarded as a single test to detect SAS. Rather, it should be regarded as one of a number of clinical indicators to consider further evaluations for SAS.

The reliability of the diagnostic data from a largescale database has occasionally been questioned [20]. Our results show that both sensitivity and specificity of the diagnostic data for SAS which requires treatment are higher than 99\%. Therefore, the data from JMDC diagnosis database could be considered reliable with both high sensitivity and specificity.

There are some limitations to this study. The JMDC database does not contain information on some risk factors for SAS such as craniofacial- and upper airway abnormalities, [21] Some of the collected lifestyle data were too simplified, e.g., smoking habit was categorized into only two groups (non-smoker vs. current smoker). Additionally, although we have a follow-up period of 1285 days, a more extended follow-up period would have allowed us to identify a larger number of cases of incident SAS. This analysis is based on a database which does not allow us to evaluate each participant's severity of SAS or detailed past medical history. Therefore, we are not able to discuss whether hemoglobin upswing is an early sign of the presence of undiagnosed SAS, or whether hemoglobin upswing presents before patients experience the signs or symptoms of SAS. Lastly, because we could not collect complementary data which also suggest the presence of SAS, e.g., polysomnography, or those using an oral appliance as a treatment, we could not identify the SAS patients who do not require CPAP or surgery. Therefore, as a further investigation, clinical studies accompanied by polysomnographic data are warranted to elucidate the reason for our findings.

Despite these limitations, this study also has several strengths. This is the first study to pay attention to the usefulness of annual health check data for the detection of SAS. This is also the first study to specifically focus on the change of hemoglobin level within the reference range. We have adjusted our analyses for the major known risk factors of SAS, and hemoglobin upswing was found not to be associated with other known risk factors. Our data on middle-aged male individuals cover a large portion of the patients who are initially diagnosed with SAS given that OSAS plateaus in patients older than 60 years of age [11]. Finally, considering that the population included in our analyses could be considered overall healthy, and that the participation rate in annual health checks in Japan is high, this result can be applied to most middle-aged men in Japan. 


\section{Conclusion}

Our data suggest that middle-aged men who experience a statistically significant increase in hemoglobin level within the reference range are more likely to be diagnosed with SAS within the next four years. Considering the situation that a large proportion of patients with SAS remain undiagnosed and that SAS can increase the risk of mortality from cardiovascular diseases, hemoglobin upswing should be paid attention to even within the reference range.

Author contributions HM and AKS were responsible for the conception and design of the study. HM analysed and interpreted the data. TS, $\mathrm{MN}$, and AKS assisted with the interpretation of the data. HM drafted the manuscript. TS, MN, and AKS critically revised the manuscript for important intellectual content. All authors approved the final version of the manuscript.

Funding Open access funding provided by Lund University. This study was funded by the Center of Innovation Program from Japan Science and Technology Agency (Grant No: JPMJCE1304), JST.

\section{Compliance with Ethical Standards}

Conflict of interest Hirotaka Miyashita declares that he has no conflict of interest. Thomas Svensson declares that he has no conflict of interest. Masahiro Nakamura declares that he has no conflict of interest. Akiko Kishi Svensson declares that she has no conflict of interest.

Ethical Approval All procedures performed in studies involving human participants were in accordance with the ethical standards of the institutional and/or national research committee and with the 1964 Helsinki declaration and its later amendments or comparable ethical standards. The study protocol of this study was approved by the Institutional Review Board of the University of Tokyo (application number: 11449).

Open Access This article is licensed under a Creative Commons Attribution 4.0 International License, which permits use, sharing, adaptation, distribution and reproduction in any medium or format, as long as you give appropriate credit to the original author(s) and the source, provide a link to the Creative Commons licence, and indicate if changes were made. The images or other third party material in this article are included in the article's Creative Commons licence, unless indicated otherwise in a credit line to the material. If material is not included in the article's Creative Commons licence and your intended use is not permitted by statutory regulation or exceeds the permitted use, you will need to obtain permission directly from the copyright holder. To view a copy of this licence, visit http://creativecommons.org/licenses/by/4.0/.

\section{References}

1. Donovan LM, Kapur VK. Prevalence and characteristics of central compared to obstructive sleep apnea: analyses from the sleep heart health study cohort. Sleep. 2016;39:1353-9. https://doi. org/10.5665/sleep.5962.

2. Young T, Evans L, Finn L, Palta M. Estimation of the clinically diagnosed proportion of sleep apnea syndrome in middle-aged men and women. Sleep. 1997;20:705-6.
3. Gottlieb DJ, Yenokyan G, Newman AB, et al. Prospective study of obstructive sleep apnea and incident coronary heart disease and heart failure: the sleep heart health study. Circulation. 2010;122:352-60. https://doi.org/10.1161/CIRCULATIO NAHA.109.901801.

4. Marin JM, Carrizo SJ, Vicente E, Agusti AGN. Long-term cardiovascular outcomes in men with obstructive sleep apnoeahypopnoea with or without treatment with continuous positive airway pressure: an observational study. Lancet (London, England). 2005;365:1046-53. https://doi.org/10.1016/S0140 -6736(05)71141-7.

5. Dempsey JA, Veasey SC, Morgan BJ, O’Donnell CP. Pathophysiology of sleep apnea. Physiol Rev. 2010;90:47-112. https://doi. org/10.1152/physrev.00043.2008.

6. Pathak R, Giri S, Karmacharya P, Aryal MR. Obstructive sleep apnea syndrome and secondary polycythemia: analysis of the nationwide inpatient sample. Sleep Med. 2015;16:205-6. https:// doi.org/10.1016/J.SLEEP.2014.09.012.

7. Japanese Law Translation [Law text] Industrial Safety and Health Act. https://www.japaneselawtranslation.go.jp/law/detai $1 / ? \mathrm{id}=3257 \& \mathrm{vm}=04 \& \mathrm{re}=02$. Accessed 17 Apr 2019

8. Ministry of Health L and W Survey on state of employees' health in 2012 (in Japanese). https://www.e-stat.go.jp/stat-search/files ?page $=1 \&$ layout $=$ datalist $\&$ toukei $=00450095 \&$ tstat $=0000010205$ $08 \&$ cycle $=0 \&$ tclass $1=000001064060 \&$ tclass $2=0000010640$ $62 \&$ stat infid $=000023628244 \& \sec$ ond $2=1$. Accessed 8 Feb 2019

9. WHO. ICD-10: international statistical classification of diseases and related health problems. 1st ed. Geneva: Switzerland; 1990.

10. Beutler E, Waalen J. The definition of anemia: what is the lower limit of normal of the blood hemoglobin concentration? Blood. 2006;107:1747-50. https://doi.org/10.1182/blood-2005-07-3046.

11. Bixler EO, Vgontzas AN, Tenhave T, et al. Effects of age on sleep apnea in men. Am J Respir Crit Care Med. 1998;157:144-8. https ://doi.org/10.1164/ajrccm.157.1.9706079.

12. Tufik S, Santos-Silva R, Taddei JA, Bittencourt LRA. Obstructive sleep apnea syndrome in the Sao Paulo epidemiologic sleep study. Sleep Med. 2010;11:441-6. https://doi.org/10.1016/j.sleep .2009.10.005.

13. Viner S, Szalai JP, Hoffstein V. Are history and physical examination a good screening test for sleep apnea? Ann Intern Med. 1991;115:356-9.

14. Myers KA, Mrkobrada M, Simel DL. Does this patient have obstructive sleep apnea? JAMA. 2013;310:731. https://doi. org/10.1001/jama.2013.276185.

15. Qaseem A, Dallas P, Owens DK, et al. Diagnosis of obstructive sleep apnea in adults: a clinical practice guideline from the American College of Physicians. Ann Intern Med. 2014;161:210. https ://doi.org/10.7326/M12-3187.

16. Boudville NC, Djurdjev O, Macdougall IC, et al. Hemoglobin variability in nondialysis chronic kidney disease: examining the association with mortality. Clin J Am Soc Nephrol. 2009;4:117682. https://doi.org/10.2215/CJN.04920908.

17. Japan Society of Ningen Dock, Criteria category (Revised on April 1, 2018), https://www.ningen-dock.jp/wp/wp-content/uploa ds/2018/06/Criteriacategory.pdf. Accessed 25 June 2020

18. Skjelbakken T, Dahl IMS, Løchen M-L. Changes in body mass index and smoking habits have a different impact on hemoglobin concentration in men and women: a longitudinal follow-up of the troms $\varnothing$ study, 1994-2002. Gend Med. 2010;7:230-9. https://doi. org/10.1016/j.genm.2010.06.006.

19. Eckardt K-U, Kim J, Kronenberg F, et al. Hemoglobin variability does not predict mortality in European hemodialysis patients. J Am Soc Nephrol. 2010;21:1765-75. https://doi.org/10.1681/ ASN.2009101017.

20. Hara K, Tomio J, Svensson T, et al. Association measures of claims-based algorithms for common chronic conditions were 
assessed using regularly collected data in Japan. J Clin Epidemiol. 2018;99:84-95. https://doi.org/10.1016/j.jclinepi.2018.03.004.

21. Young T, Skatrud J, Peppard PE. Risk factors for obstructive sleep apnea in adults. JAMA. 2004;291:2013. https://doi.org/10.1001/ jama.291.16.2013.
Publisher's Note Springer Nature remains neutral with regard to jurisdictional claims in published maps and institutional affiliations. 